\title{
Avaliação de um novo implante intra-orbitário: resultados preliminares
}

\author{
Evaluation of a new intraorbital implant:preliminary results
}

Thierry Malet $^{1}$

Cláudio Asperti Spera²

Ana Paula Ximenes Alves ${ }^{3}$
${ }^{1}$ Oftalmologista, chefe do setor de Oculoplástica da Clinique Monticelli, Marselha - França.

${ }^{2}$ Oftalmologista, pós-graduando pela Universidade $\mathrm{Fe}$ deral de São Paulo - UNIFESP.

${ }^{3}$ Oftalmologista, professora substituta da Disciplina de Oftalmologia da Faculdade de Medicina da Universidade Federal do Ceará, doutorado em Oftalmologia pela Universidade de São Paulo - Ribeirão Preto.

Endereço para correspondência: Rua Osvaldo Cruz, 1000/1504 - Fortaleza (CE) CEP 60125-150.

E-mail: apxalves@hotmail.com

Recebido para publicação em 04.12.2000 Aceito para publicação em 11.09.2002

\begin{tabular}{|l|}
\hline \multicolumn{1}{|c|}{ RESUMO } \\
\hline Objetivo: Apresentar novo tipo de implante orbitário, com formato original, \\
o modo de utilização e os resultados clínicos preliminares. Métodos: Os \\
autores descrevem a técnica cirúrgica utilizada para a inserção do novo \\
implante em 22 pacientes, como implante primário ou secundário. Os \\
pacientes foram avaliados de forma prospectiva quanto aos seguintes \\
aspectos: cosmético (grau de sulco suprapalpebral e enoftalmia), mobilida- \\
de, centragem e volume do implante. O tempo de seguimento variou de 3 \\
a 15 meses (média 1 ano). Resultados: Observou-se resultado cosmético \\
satisfatório, sem casos de enoftalmia ou de sulco suprapalpebral importan- \\
te, bem como boa mobilidade das próteses adaptadas sobre o novo \\
implante. Não houve casos de infecção, migração ou extrusão do implante. \\
Conclusão: Os resultados pós-operatórios com o novo implante são \\
comparáveis aos implantes com pinos externos. Contudo um estudo \\
multicêntrico, com maior tempo de controle pós-operatório é necessário, \\
para avaliação mais acurada das complicações potenciais. \\
\hline
\end{tabular}

Descritores: Implantes orbitários; Órbita/cirurgia; Materiais biocompatíveis

\section{INTRODUÇÃO}

Após a indicação cirúrgica da enucleação/evisceração, a aceitação do "olho artificial" pelo paciente vai depender fundamentalmente de um bom resultado pós-operatório, tanto no plano cosmético como na mobilidade da prótese ocular. Para a melhora da cosmética da cavidade anoftálmica, a primeira introdução de um implante intra-orbitário, de vidro, com o objetivo de aumentar o volume da cavidade foi descrita em $1885^{(1)}$. As taxas de extrusão eram extremamente altas, chegando a 93\%. O material utilizado para a confecção dos implantes foi sendo modificado, até chegarmos aos implantes bioreativos como a hidroxiapatita, o polietileno poroso e a biocerâmica. Estes implantes possuem microporos de 200 a $500 \mu \mathrm{m}$ que favorecem a proliferação vascular, aumentando a mobilidade e diminuindo os riscos de infecção, de migração e de extrusão.

Com o objetivo de melhorar a mobilidade, propôs-se ${ }^{(2)}$, em 1947, a fixação de um pino, em um segundo tempo operatório, ao implante intraorbitário já presente, sobre o qual se encaixa a prótese. Observa-se, contudo, que as taxas de complicações com pinos é grande. Já foram descritas ${ }^{(3-4)}$ taxas de $37 \%$ de extrusão com pinos em titânio e $27 \%$ de extrusão com pinos combinados de titânio e hidroxiapatita. As altas taxas de extrusão devem-se ao fato de que existem diferentes forças de atrito entre os materiais: entre o implante intra-orbitário e o pino, e entre o pino e a prótese externa, que acabam por favorecer a extrusão deste pino $^{(5)}$. 
Ao nosso ver, o implante ideal deve fazer parte integrante da órbita para diminuir as chances de extrusão. Seu volume deve ser adaptado ao tamanho da órbita para diminuir as complicações cosméticas. Ele deve ainda estar em íntimo contato com a prótese externa para aumentar a mobilidade.

Procurando buscar estas características, desenvolvemos conjuntamente com o Laboratório $\mathrm{FCI}^{\circledast}$ um novo implante intra-orbitário, composto de uma porção posterior com 16 a 20 $\mathrm{mm}$ de diâmetro e de uma cúpula anterior de $10 \mathrm{~mm}$ de diâmetro. A intenção é de que a prótese se encaixe à cúpula anterior, que lhe transmitirá os movimentos do côto, substituindo os pinos tradicionais. As duas partes formam um só implante monobloco, não havendo separação entre elas. Não há nenhuma espécie de pino dentro do implante. Como matéria prima, é fabricado em material poroso, em biocerâmica, construído segundo as normas européias de produção.

As cerâmicas são materiais inorgânicos constituídos por silicatos ou óxidos metálicos preparados a altas temperaturas, que têm sido utilizados, em diversas áreas da Medicina há, pelo menos, 30 anos $^{(6-7)}$. O sucesso da biocerâmica como implante para cavidades anoftálmicas já foi demonstrado por outros autores ${ }^{(5,8)}$. Na foto 1 podemos observar o perfil do novo implante através da tomografia computadorizada.

\section{OBJETIVOS}

Avaliar o aspecto estético e funcional das cavidades receptoras do novo implante, bem como as complicações relacionadas a ele ou à técnica cirúrgica utilizada para a sua implantação.

\section{MÉTODOS}

Foram incluídos nesse estudo 22 olhos de 22 pacientes,

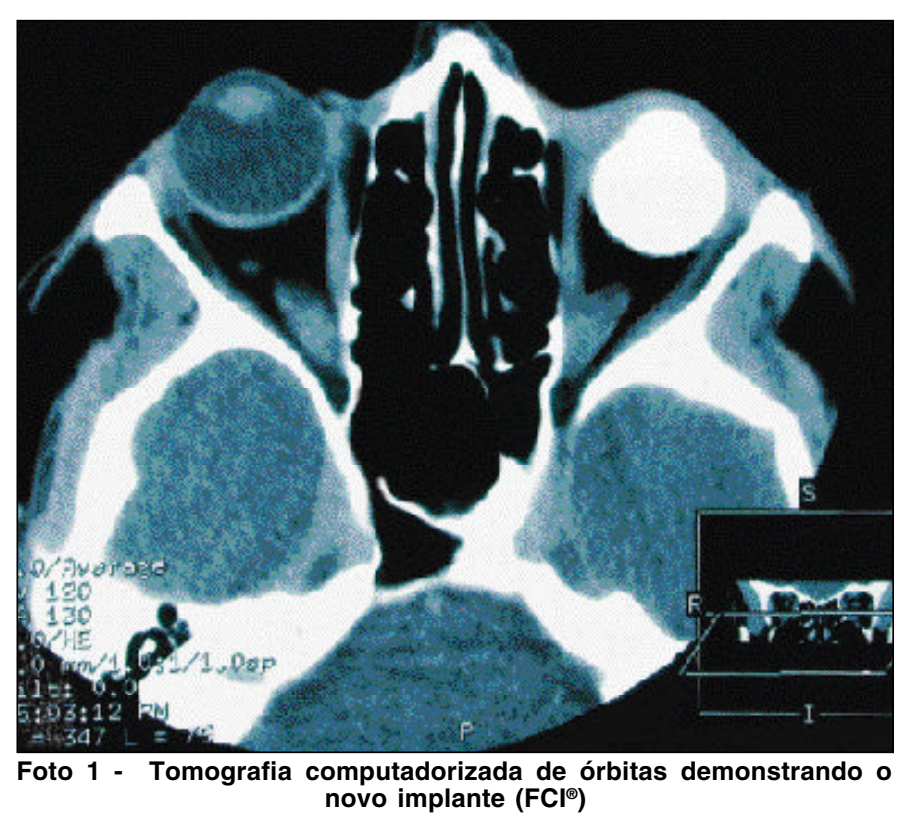

sendo estes 10 mulheres e 12 homens, apresentando idade média de 49,18 anos (14 a 86 anos). Na tabela 1 encontram-se as patologias pré-operatórias dos pacientes, submetidos à evisceração ou enucleação.

A enucleação foi realizada em $18 \%$ dos casos e a evisceração, nos $82 \%$ restantes. Foram utilizados implantes com diâmetro posterior de $16 \mathrm{~mm} \mathrm{em} \mathrm{4,6 \%} \mathrm{dos} \mathrm{casos,} \mathrm{de} 18 \mathrm{~mm}$ em $63,6 \%$ dos casos e de $20 \mathrm{~mm}$ em $31,8 \%$ dos casos.

Em relação à mobilidade pré-operatória, dividimos os pacientes em grupo A e grupo B. No grupo A, os pacientes apresentavam mobilidade excelente em $50 \%$ dos casos e boa nos 50\% restantes. Nesses pacientes, o número médio de cirurgias prévias era de 0,8 , sendo que $43,7 \%$ destes submeteram-se a cirurgias prévias do segmento anterior e $31,2 \%$ a cirurgias do segmento posterior.

No grupo B, a mobilidade pré-operatória foi predominantemente regular (em 66,6\%) ou ruim (em 16,7\%) e numa minoria (16,7\% dos casos), foi excelente. Não houve pacientes com mobilidade pré-operatória considerada boa neste grupo. $\mathrm{O}$ número médio de cirurgias prévias nesse grupo foi de 1,66 ; sendo que $30 \%$ destes submeteram-se a cirurgias do segmento anterior e 50\%, cirurgias do segmento posterior.

\section{TÉCNICA CIRÚRGICA}

\section{Enucleação}

Foi realizada a técnica clássica para enucleação ${ }^{(9)}$. O implante foi introduzido na órbita tomando-se cuidado com a correta centragem da cúpula anterior, que foi recoberta em 1 caso, com fáscia lata, e em 4 outros, com fáscia temporal superficial. Os músculos extra-oculares foram suturados ao tecido utilizado para o recobrimento, ao nível da base da cúpula anterior, e em seguida, suturados entre si conforme demonstrado na figura 1. Deste modo o implante encontra sua posição natural dentro do cone muscular. Finalmente, foi realizada uma sutura cuidadosa em dois planos: plano tenoniano e plano conjuntival. Em todos os pacientes foi adaptado um molde escleral fenestrado.

\section{Evisceração}

Foi realizada a técnica de evisceração com auto-enxerto escleral $^{(10)}$. Após peritomia e dissecção da conjuntiva e Tenon, os músculos extra-oculares foram desinseridos e procedeu-se à

\begin{tabular}{|c|c|}
\hline \multicolumn{2}{|c|}{ EVISCERAÇÃO - 17 casos } \\
\hline Trauma ocular & $47 \%$ \\
\hline Descolamento de retina & $17 \%$ \\
\hline Glaucoma & $18 \%$ \\
\hline Phithisis bulbi & $18 \%$ \\
\hline \multicolumn{2}{|c|}{ ENUCLEAÇÃO - 5 casos } \\
\hline Melanoma maligno & $80 \%$ \\
\hline Endoftalmite & $20 \%$ \\
\hline
\end{tabular}




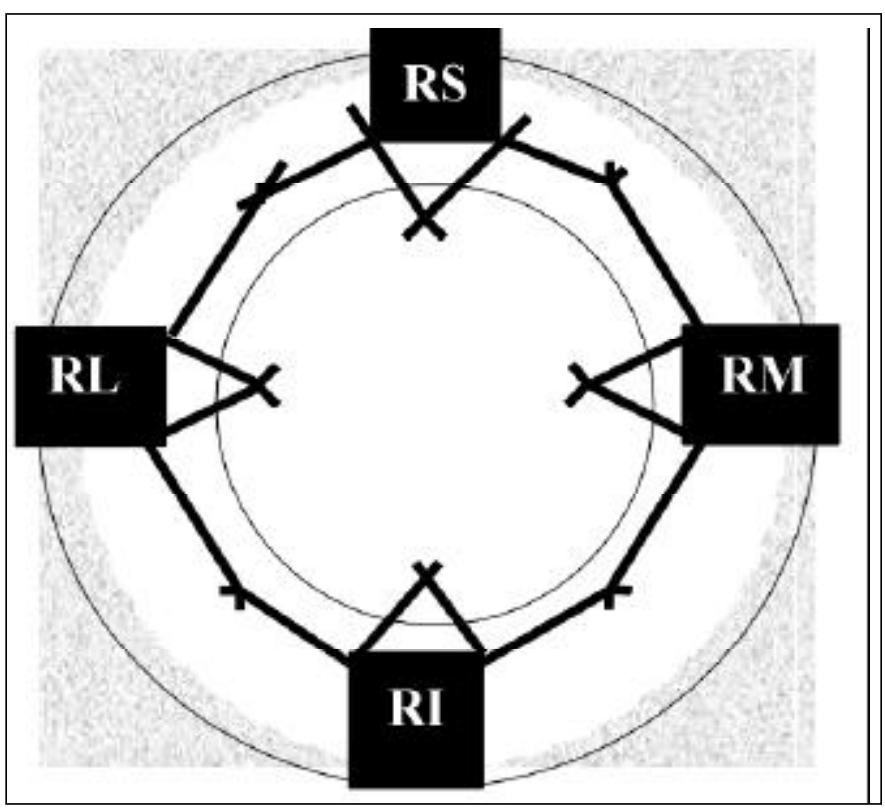

Figura 1 - Diagrama representativo da técnica cirúrgica para sutura dos músculos oculomotores na base da cúpula anterior do implante, tanto para enucleação como evisceração $(\mathrm{RS}=$ reto superior, $\mathrm{RI}=$ reto inferior, $\mathbf{R M}=$ reto medial, $\mathbf{R L}=$ reto lateral)

exérese corneana e ao esvaziamento do globo. Foi retirado o enxerto escleral entre 9 e 12 horas e o implante foi introduzido na cavidade escleral restante, tomando-se cuidado com a centragem. O enxerto foi utilizado para o recobrimento da cúpula do implante, sobre o qual foram suturados os músculos extraoculares, ao nível da base da cúpula anterior. Em seguida, os músculos foram suturados entre si.

O cuidado em desinserir os músculos extra-oculares, reinseri-los à cúpula do implante e suturá-los entre si foi necessário para favorecer uma boa centragem do implante, situando-o no cone muscular, e para avançar as inserções musculares, que se tornaram frouxos após a retirada do enxerto escleral.

Finalmente, foi realizada uma sutura cuidadosa em dois planos: plano tenoniano e plano conjuntival. Em todos os pacientes foi adaptado um molde escleral fenestrado

\section{Avaliação Pós-operatória}

A avaliação dos pacientes foi realizada por dois cirurgiões oculoplásticos, um protético e pelo próprio paciente. Foram observados: a cosmética global do paciente e a qualidade do núcleo orbitário (volume, centragem e mobilidade).

$\mathrm{O}$ aspecto cosmético foi classificado em (exame da cavidade com a prótese):

- Excelente, quando havia máxima similaridade entre o olho restante e o lado da prótese, com sulco palpebral bem preenchido e ausência de enoftalmia;

- Bom, quando se notava ligeira disparidade entre os dois lados, às custas de pequeno sulco palpebral;

- Médio, quando havia sulco palpebral moderado, sem enoftalmia;
- Regular, quando havia sulco palpebral evidente e certo grau de enoftalmia;

- Ruim, quando havia enoftalmia evidente.

$\mathrm{O}$ volume foi classificado em (exame da cavidade sem a prótese):

- Bom, quando se percebia bem o abaulamento do implante na cavidade;

- Médio, quando o implante era percebido mas não era protuberante;

- Ruim, quando o implante era pobremente percebido;

- Pobre, quando não parecia haver implante orbitário.

A centragem do implante foi conferida através do exame da cavidade sem a prótese, estando o paciente fixando em frente e classificada em centrada ou não.

A mobilidade foi classificada em (exame da cavidade com a prótese):

- Excelente, quando as ducções eram similares a de um olho normal;

- Boa, quando havia certa perda das ducções extremas;

- Média, quando havia perda evidente de uma das ducções básicas (supra, infra, levo ou dextro);

- Regular, quando havia perda evidente de duas das 4 ducções;

- Ruim, quando havia perda evidente de 3 ou 4 ducções.

\section{RESULTADOS}

Na foto 2 observamos o aspecto de 6 meses de uma cavidade anoftálmica com o novo implante e pode-se notar a pequena protuberância dada pela cúpula anterior.

Apresentamos aqui o último controle de todos os pacientes, média de 1 ano de controle pós-operatório ( 3 a 15 meses). Quanto ao aspecto cosmético global (Tabela 2), ele foi avaliado como sendo excelente ou bom em $77,3 \%$ dos casos, e médio

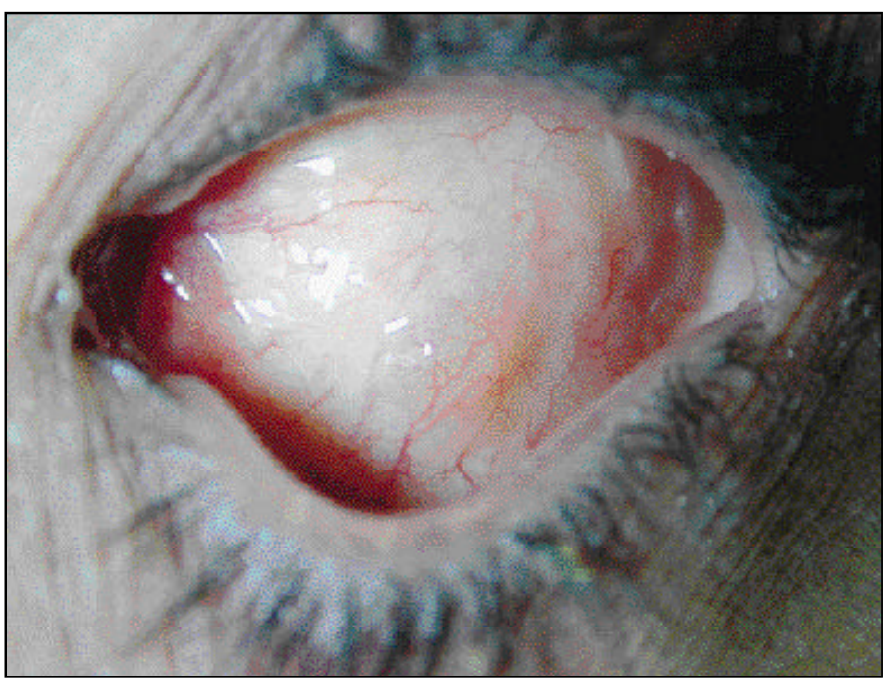

Foto 2 - Aspecto externo do implante $\left(\mathrm{FCl}^{\oplus}\right)$ com 6 meses de controle. 

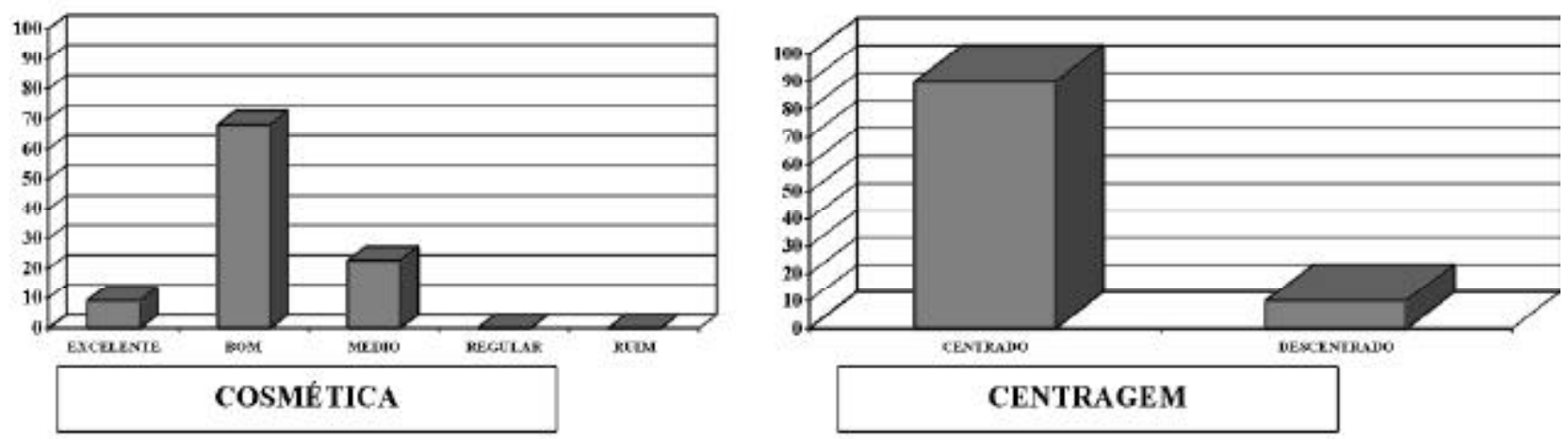

Tabela 2 - Aspecto cosmético e centragem pós-operatória do novo implante (valores em porcentagem)

em $22,7 \%$. Estes últimos apresentavam sulco palpebral superior moderado, porém sem enoftalmia.

Em $90 \%$ dos pacientes, observamos uma centragem correta do núcleo. Nos $10 \%$ restantes, o núcleo estava descentrado (Tabela 2).

Em relação ao volume orbitário pós-operatório, ele foi avaliado como bom ou médio em $80 \%$ dos casos e como ruim ou pobre em 20\% (Tabela 3).

Quanto à mobilidade pós-operatória, observamos que os pacientes do grupo A (mobilidade pré-operatória excelente em $50 \%$ dos casos e boa nos $50 \%$ restantes) mantiveram mobilidade excelente ou boa, assim como os do grupo B (mobilidade préoperatória regular ou ruim em $83,3 \%$ dos casos) mantiveram mobilidade média ou regular (Tabela 4). Não houve pacientes do grupo A apresentando mobilidade pós-operatória média, regular ou ruim. No grupo B, não houve pacientes com mobilidade pós-operatória considerada excelente, boa ou ruim.

Não se observou, nesta série, nenhum caso de infecção, migração ou extrusão do implante.

\section{DISCUSSÃO}

A bibliografia sobre implantes intra-orbitários mostra a busca constante de implantes bem tolerados e capazes de

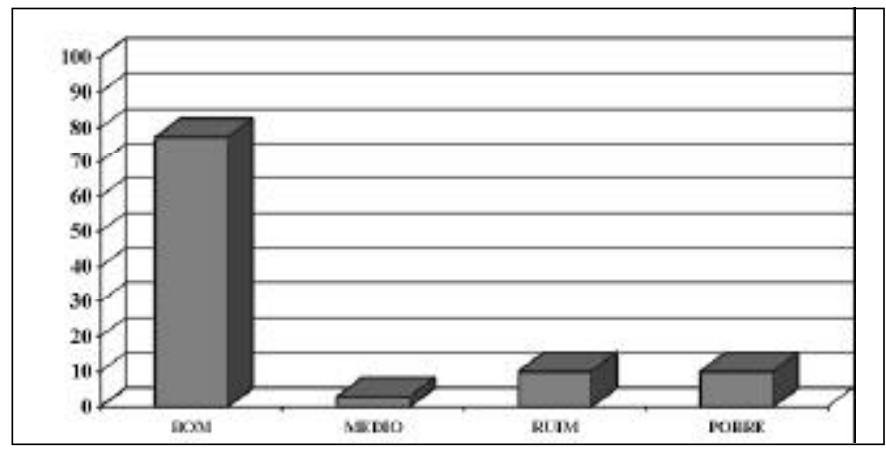

Tabela 3 - Volume orbitário pós-operatório das cavidades portadoras do novo implante (valores em porcentagem) assegurar boa mobilidade à prótese ${ }^{(5,11-14)}$. A presente série de casos não teve como objetivo avaliar a biocompatilidade da biocerâmica, material que já demonstrou ser bem tolerado e comparável a outros materiais ${ }^{(6)}$. Ao invés disso, o estudo apontou que esse novo conceito em implante intra-ocular pode ser uma alternativa que oferece aumento satisfatório do volume orbitário aliado a boa mobilidade.

Enquanto um implante normal, esférico, de $18 \mathrm{~mm}$ de diâmetro, apresenta um volume de $3,1 \mathrm{cc}^{(15)}$, o novo implante apresenta um volume total de $3,31 \mathrm{cc}$, graças à cúpula anterior que aumenta o volume implantado. Este aumento de volume extra ajuda a melhorar a cosmética dos pacientes e a diminuir a incidência de sulco palpebral superior profundo.

Quanto à mobilidade, obtivemos resultados comparáveis aos obtidos com implantes dotados de pinos externos ${ }^{(16-17)}$, porém superiores aos obtidos com implantes tradicionais. A presença da cúpula anterior favorece a moldagem da prótese externa, que torna-se fina e pode se apoiar na cúpula, aumentando dessa forma a mobilidade global do conjunto implante-prótese. Contudo, deve-se tomar cuidado com o volume adicional fornecido pela cúpula, pois existe menos espaço para a confecção da câmara anterior da prótese. Portanto a câmara anterior precisa ser afinada e a adaptação da prótese deve ser feita por um protético que conheça as novas características desse implante.

Observamos que pacientes que apresentaram boa mobilidade pós-operatória (grupo A), já a apresentavam no préoperatório. Eram pacientes que tinham sido operados poucas vezes anteriormente (média de 0,8 cirurgias) e principalmente de cirurgias do segmento anterior. Com a colocação do novo implante, conseguimos manter essas características. Da mesma forma os pacientes que apresentaram resultados ruins no pós-operatório (grupo B), já apresentavam no exame pré-operatório uma mobilidade ocular deficitária. São pacientes que foram submetidos a um maior número de cirurgias do que o grupo A (média de 1,66 cirurgias), e principalmente do segmento posterior. Parece haver um consenso que pacientes operados da retina e vítreo, cujos músculos oculomotores tenham sido manipulados amplamente, apresentam mobilidade reduzida no pós-operatório( ${ }^{(18-19)}$. 


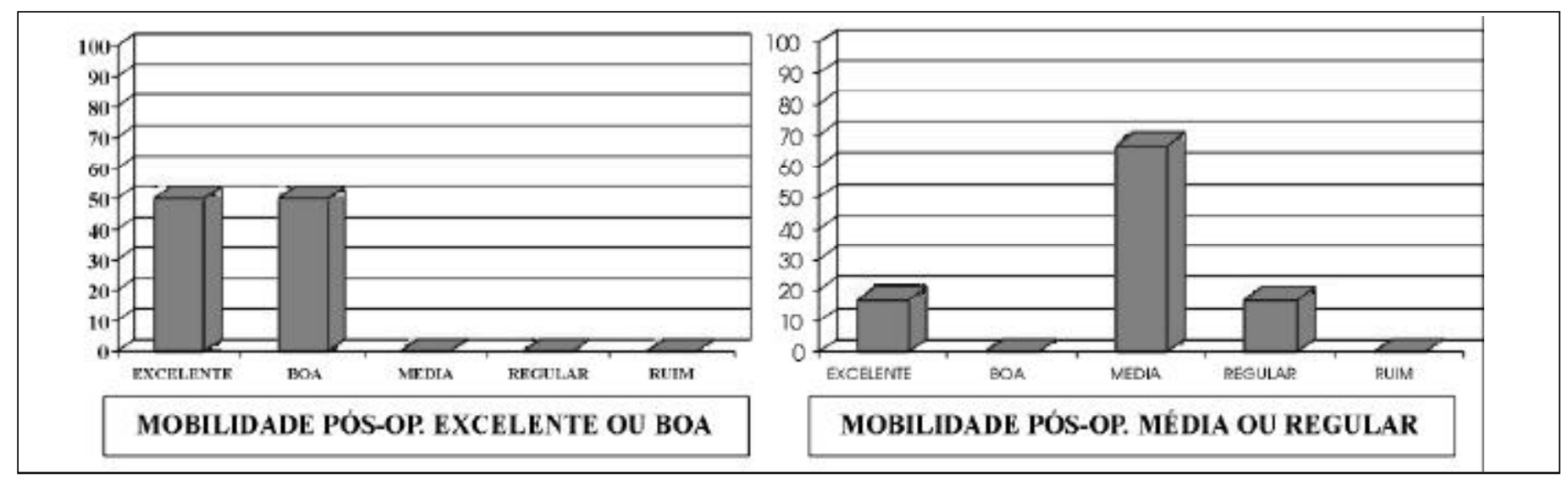

Tabela 4 - Mobilidade pós-operatória do implante, segundo a mobilidade pré-operatória (valores em porcentagem)

Uma particularidade em relação ao novo implante foi a necessidade de um cuidado especial com o recobrimento da cúpula anterior (por ser mais proeminente do que os implantes tradicionais). Para tanto, foi utilizada a técnica de evisceração com auto-enxerto escleral ${ }^{(10)}$, que permite a mobilização da esclera para o recobrimento da cúpula anterior, deixando a porção posterior do implante exposta e passível de vascularização. Assim como no estudo original, realizado por Tunc et col., essa técnica mostrou-se segura e relativamente simples de ser praticada.

Outra particularidade foi a preocupação com a centragem, já que dificilmente seria possível adaptar uma boa prótese diante de uma cúpula rodada. Para tanto, os cuidados em reinserir os músculos (inclusive nas eviscerações) à cúpula do implante e suturá-los entre si parecem ter sido suficientes, pois a centragem do implante foi considerada correta na maioria dos casos.

Concluindo, o novo implante, que foi criado como alternativa para os implantes com pinos externos, mostrou mobilidade comparável à daqueles, porém com menos riscos. Além disso, aliou as vantagens de:

- aumento do volume implantado, favorecendo a cosmética global,

- formato original, que favorece a confecção de próteses mais finas,

- porosidade, que propicia uma boa integração aos tecidos orbitários.

Para a sua colocação, foi utilizada uma nova técnica de sutura dos músculos oculomotores, para a segurança de uma boa centragem. Um estudo multicêntrico, com maior tempo de controle pós-operatório, se faz necessário para avaliar as possíveis complicações e determinar a real taxa de extrusão.

\section{ABSTRACT}

Purpose: To describe a new orbital implant, with an original shape, the surgical technique adapted for its implantation and preliminary results. Methods: The authors describe the surgical technique performed in 22 patients, either as primary or secondary implants. The patients were evaluated in a prospective way to cosmesis (degree of upper lid sulcus and enophthalmos), implant mobility, volume and centralization. Follow-up ranged from 3 to 15 months (average 1 year). Results: A good cosmetic result without cases of severe upper lid sulcus or enophthalmos and a good prosthesis mobility were observed. There was no implant infection, migration or extrusion. Conclusions: We conclude that the clinical results with the new implant can be compared to the usual coupled ones, but a multicentric study, with longer follow-up is necessary, for a better evaluation of its potential complications.

Keywords: Orbital implants, Orbit/surgery; Biomaterials, compatible

\section{REFERÊNCIAS}

1. Mules PH. Evisceration of the globe with artificial vitreous. Trans Ophthalmol Soc U K, 1885;5:200-6.

2. Cutler ML. A positive contact ball and ring implant after enucleation. Arch Ophthalmol 1947;37:73-81.

3. Jordan DR. Complications associated with pegging hydroxyapatite implants. In: European Society of Ophthalmic Plastic and Reconstructive Surgery. ESOPRS Meeting; 1998 Out 3. Budapest, Hungria; 1998. p.40.

4. Jordan DR. Complications with pegging hydroxyapatite implants. In: American Society of Ophthalmic Plastic and Reconstructive Surgery. ASOPRS Meeting; 1999 Nov 7. New Orleans, USA; 1999. p.35.

5. Soares EJC, França VP, Wykrota L, Stumpf S. Clinical evaluation of a new bioceramic ophthalmic implant. Orbit 1995;14:137-46.

6. Cini L, Sandrolini S, Paltrinieri M, Pizzoferrato A, Trentani C. Bioceramic materials for replacement purposes. Preliminary note. Chir Organi Mov 1972;60:423-30.

7. Canella P. Reflexions on the use of bioceramic material in orthopedic surgery. Chir Organi Mov 1978;64:665-70.

8. Jordan DR, Mawn LA, Brownstein S, McEachren TM, Gilberg SM, Hill V et al. The bioceramic orbital implant: a new generation of porous implants. Ophthal Plast Reconstr Surg 2000;16:347-55.

9. Mustarde JC. Repair and reconstruction in the orbital region. $2^{\text {nd }}$ ed. New York: Churchill Livingstone; 1991. p.331-40.

10. Tunc Z, Salvanet-Bouccara A, Fournier G. Nouvelle approche chirurgicale dans la technique d'éviscération avec utilisation d'une autogreffe sclérale. Ophtalmologie 1997;1:352-3.

11. Pórez-Blázquez GJ, González-Santos R, Acosta Díaz L, Solano Bravo ME, Oliva Acosta JL, Rodríguez Pérez JL Hidroxiapatita porosa HAP-200 como 
bio implante esférico integrado en el anoftalmos quirúrgicos. Rev Cuba Oftalmol 1998;11:5-13.

12. Schellini SA, Silva MRBM, Hata MM. Uso da malha tubular de poliester como envoltório do implante de cavidade. Rev Bras Oftalmol 1998;57:207-10.

13. Queiroz Neto L, Betinjane JA, Salazar SRA. Implante de Dracon. Arq Inst Penido Burnier 1992;34:41-4.

14. Soares EJC. Tratamento das deformidades órbito-palpebrais das cavidades anoftálmicas: 20 anos de experiência. Arq Bras Oftalmol 1990;53:126-31.

15. Soares EJC, Dantas RRA, Marback R, Matayoshi S, França VP. Cavidades anoftálmicas. In: Soares EJC, Moura EM, Gonçalves JOR. Cirurgia plástica ocular. São Paulo: Roca; 1997. p.327-68.
16. Coden DJ. Surgical technique for a novel titanium motility/support peg system. In: American Society of Ophthalmic Plastic and Reconstructive Surgery. SOPRS Meeting; 1999 Nov 7; New Orleans, USA; 1999. p.36.

17. Kikkawa DO. Prosthetic motility in pegged versus unpegged integrated poros orbital implants. In: American Society of Ophthalmic Plastic and Reconstructive Surgery. ASOPRS Meeting; 1999 Nov 7; New Orleans, USA; 1999. p.33.

18. de Gottrau P, Holbach LM, Naumann GO. Clinicopathological review of 1146 enucleations (1980-90). Br J Ophthalmol 1994;78:260-5.

19. Meltzer MA. Complications of enucleation and evisceration: prevention and treatment. Int Ophthalmol Clin 1992;32:213-33. 